\title{
National Blood Cancer Experts Provide Updates on CAR T-Cell Therapy, Leukemia, Lymphoma, and Myeloma; New NCCN Guidelines for Systemic Mastocytosis Now Available
}

Hundreds of blood cancer experts met in New York City to discuss how treatment options have transformed over the past year, as part of the NCCN 13th Annual Congress: Hematologic Malignancies. The congress was hosted by NCCN, and moderated by cochairs Andrew D. Zelenetz, MD, PhD, Medical Director, Quality Informatics, Memorial Sloan Kettering Cancer Center and Ranjana H. Advani, MD, Saul Rosenberg Professor of Lymphoma, Stanford University Medical Center. The congress featured a keynote session on CAR T-cell therapy and educational sessions on treatment updates for 14 different cancer types, some of which include acute myeloid leukemia (AML), chronic lymphocytic leukemia (CLL), chronic myeloid leukemia (CML), Hodgkin lymphoma, multiple myeloma (MM), and myeloproliferative neoplasms (MPN), as well as the management of bone health in patients with blood cancers.

"We are devoted to making sure that providers who serve people with cancer are knowledgeable about promising new cancer treatments," said Robert W. Carlson, MD, Chief Executive Officer, NCCN. "This congress and our frequently updated NCCN Guidelines allow us to share the best new evidence and to offer insights into new care recommendations from our expert panelists."

"Numerous advances have been made in the biology and treatment of hematological malignancies in 2018," said Dr. Zelenetz. "Today, an unprecedented number of novel drugs have been approved or are in the developmental pipeline. Many of these drugs act through entirely novel mechanisms of action and target particular molecularly defined subsets of disease."

Dr. Advani agreed: "The complexity of integrating risk assessment, optimal therapy, molecular profiles, surveillance and the continuum of care concepts across hematological malignancies has challenged the practitioner to keep abreast of an evolving diagnostic and treatment landscape, which is best addressed by ongoing comprehensive educational opportunities and utilization of guidelines."

The day-and-a-half long event began with a series of keynote addresses on milestones in CAR T-cell therapy from Frederick L. Locke, MD, Moffitt Cancer Center, plus a closer look at using CAR T-cell therapy in lymphomas and leukemia from Reem Karmali, MD, MS, Robert H. Lurie Comprehensive Cancer Center of Northwestern University, and Patrick A. Brown, MD, The Sidney Kimmel Comprehensive Cancer Center at Johns Hopkins, respectively. Richard M. Stone, MD, Dana-Farber Cancer Institute, followed with an address about considerations for the use of novel agents in AML.

The second day focused on patient case studies and panel discussions, and included the additional faculty:

- John N. Allan, MD, Weill Cornell Medical

- Philippe Armand, MD, PhD, Dana-Farber Cancer Institute

- Natalie S. Callander, MD, University of Wisconsin Carbone Cancer Center

- Azeez Farooki, MD, Memorial Sloan Kettering Cancer Center

- Reem Karmali, MD, MS, Robert H. Lurie Comprehensive Cancer Center of Northwestern University

- Shaji K. Kumar, MD, Mayo Clinic Cancer Center

- Michael J. Mauro, MD, Memorial Sloan Kettering Cancer Center

- Animesh Pardanani, MBBS, PhD, Mayo Clinic Cancer Center

- Emily N. Patterson, AGACNP-BC, Memorial Sloan Kettering Cancer Center

- Jerald P. Radich, MD, Fred Hutchinson Cancer Research Center/Seattle Cancer Care Alliance

Cont. on page xxiv. 
Cont. from page xxii.

\section{- Eunice S. Wang, MD, Roswell Park Comprehensive Cancer Center}

Enduring webcasts from the NCCN 13th Annual Congress: Hematologic Malignancies will be available online at education.nccn.org starting on November 1 .

This congress took place just after the publication of a brand new NCCN Guidelines for Systemic Mastocytosis, the most common form of mastocytosis diagnosed in adults. Systemic mastocytosis mainly results from the accumulation of abnormal mast cells (a type of white blood cell) in one or more extracutaneous organs (with or without skin involvement). The new guidelines were developed by the NCCN Guidelines Panel for MPN, and include recommendations for the diagnosis and management of patients with all variants of systemic mastocytosis.

Looking ahead, the NCCN 2019 Annual Conference will take place March 21-23, 2019, in Orlando, Florida. The NCCN 2019 Annual Congress: Hematologic Malignancies is scheduled for September 27-28, 2019, in San Francisco, California.

\section{NCCN Summit Explores How Current Health Policies Help and/or Hinder the Coordination of High-Quality Cancer Care Delivery in the United States}

NCCN hosted a summit exploring the current health policy landscape in the United States, and what it means for the delivery and coordination of high-quality cancer care. The NCCN Oncology Policy Summit: Policy Challenges and Opportunities to Address Changing Paradigms in Cancer Care Delivery took place at the National Press Club in Washington, DC. Experts from across the cancer care continuum discussed how current policies help or hinder optimal care delivery, with a focus on innovation, value-based models, the changing cancer care workforce, and coordination of care.

"NCCN encourages the creation and implementation of policies that enable better access to evidence-based cancer treatment," said Robert W. Carlson, MD, Chief Executive Officer, NCCN. "The experts on the NCCN Guidelines Panels weigh the best available evidence before recommending the best approaches for treating individuals with cancer. Healthcare policies should facilitate the delivery of these best approaches, not hinder them."

Cancer care needs to continuously evolve with advances in innovation and changes in related care delivery to ensure access and maximize quality and outcomes; related policies in the United States do not always keep pace with these needs. In response, NCCN convened a working group to suggest short- and long-term policy strategies for implementing best practices for overcoming the disconnect. Warren Smedley, MSHA, MSHQS, UAB Cancer Community Network, University of Alabama at Birmingham Comprehensive Cancer Center, and Leonidas C. Platanias, MD, PhD, Robert H. Lurie Comprehensive Cancer Center of Northwestern University, presented the group's findings during the summit.

"NCCN recognizes the need to provide increasingly higher value care to our patients by continuously striving to improve care, streamline processes, and reduce unnecessary utilization," explained Smedley. "To achieve this, we are emphasizing the need for greater transparency and availability of patient care data through the sharing and exchange of claims data from CMS and private payers, as well as greater functionality and interoperability for electronic health record systems."

Smedley continued: "Effective value-based cancer care requires a holistic, team-based approach. Yet, current reimbursement practices do not typically recognize the value of the extended care team. With the looming shortage of physicians, we need to develop team-based payment models that support a broader skillset and greater diversity on the care team." 
Lee Newcomer, MD, MHA, who formerly oversaw oncology for UnitedHealthcare, gave a keynote address on how potentially negative consequences can arise out of good intentions.

"Society wants effective cancer therapies to be affordable," said Dr. Newcomer. "Wellintended policies to guarantee access to pharmaceuticals have allowed prices to skyrocket. The tragedy of these policies is that those high prices are restricting access."

Drs. Newcomer and Platanias also participated in a panel discussion on innovative treatment and coordination of care, which also featured:

- Yelak Biru, Cancer survivor, Patient champion

- Eric Konnick, MD, MS, University of Washington

- Ray Page, DO, PhD, The Center for Cancer and Blood Disorders in Fort Worth

- Mary Pinder-Schenck, MD, Merck

- Amy B. Porter-Tacoronte, MBA, The Sidney Kimmel Comprehensive Cancer Center at Johns Hopkins

Another panel discussion, focused on delivery high-quality cancer care under valuebased payment models, included Drs. Newcomer and Smedley as well as:

- Christian G. Downs, MHA, JD, Association of Community Cancer Centers

- Andrea E. Ferris, MBA, LUNGevity

- John O'Shea, MD, The Heritage Foundation

- Robert S. Saunders, PhD, Duke-Margolis Center for Health Policy

Both panels were moderated by Clifford Goodman, PhD, of The Lewin Group.

NCCN will host its next policy summit on December 10th. It will focus on Patient Advocacy and Equity, and feature Lynette Bonar, RN, MBA, CEO, Tuba City Regional Health Care Corporation, who will give a keynote address on providing cancer care for the Navajo Nation. Visit NCCN.org/policy for more information and join the conversation online with the hashtag \#NCCNPolicy.

\section{NCCN and Lilly Support Quality Improvements for Gastric Cancer Care}

The NCCN Oncology Research Program (ORP) and Eli Lilly and Company (NYSE: LLY) are collaborating to seek proposals for improving care for gastric and gastroesophageal junction (GEJ) cancer patients in the United States. Healthcare institutions, professional organizations, advocacy groups, and other organizations working to improve the quality of care for this patient population are invited to apply.

"We're particularly interested in projects that address gaps in clinical care through interventions that are easily scalable, reproducible, and implementable across various diverse oncology practice settings," said Susan Most, RN, MBA, Director, Clinical Operations, NCCN ORP. "This request for proposals seeks to identify and ameliorate some of the challenges faced by people with gastric/GEJ cancer and their healthcare providers. We hope the results from these projects can be quickly disseminated to other practices and settings to rapidly improve delivery of cancer care."

There are multiple factors that contribute to the complexity of treating gastric cancer, which is also known as stomach cancer. Difficulties include managing treatment and disease-related side effects, nutritional concerns, psychological distress and anxiety, employment re-entry, and financial burden. Additionally, it's crucial to make sure patients and caregivers are provided with the resources and education needed to ensure informed, shared decision-making. 
Cont. from page xxv.
"Gastric cancer is a leading cause of cancer-related deaths globally, and Lilly is deeply committed to patients with this aggressive disease," said Maura Dickler, MD, Vice President of Late Phase Development, Lilly Oncology. "We are proud to collaborate with NCCN in furthering our efforts to improve care and provide support for people living with gastric and GEJ cancer."

The Request for Proposals (RFP) will focus on the following areas of emphasis:

1. Opportunities for innovative programs, tools, technologies and clinical pathways or platforms to improve:

- Adherence to evidence-based medicine guidelines, including, when appropriate, choosing systemic regimens based on current, best practices for the treatment of gastric/GEJ cancer.

- Collaboration between comprehensive cancer centers and community hospitals.

- Patient safety during treatment and patient adherence to treatment.

- Holistic and integrative or evidence-based complementary medicine support programs around nutrition, physical activity, psychosocial counseling, financial counseling, and other areas of need.

- Multidisciplinary collaborations within the community setting, including partnerships with businesses and foundations.

2. Opportunities to address gaps in clinical practice (including clinician, patient, healthcare system, financial, and organizational factors) through improving:

- Healthcare professional competencies (excluding requests for continuing medical education grants).

- Healthcare system-based management.

- Patient oriented solutions.

- External factors that impact patient care and clinical outcomes.

The NCCN ORP — which supports scientifically meritorious research studies at NCCN Member Institutions and other cancer centers-will oversee application review and evaluation. A committee led by NCCN and including a medical representative from Lilly will determine which proposals will receive funding. Funding will be provided by Lilly.

The RFP letter-of-intent deadline is October 24, 2018. Visit NCCN.org/ORP for more information.

\section{New Guidelines Provide Vital Information for Women Diagnosed With the Most Common Type of Gynecologic Cancer}

While the number of deaths from most cancers is on the decline in the United States, it is rising for the most common type of uterine cancer-endometrial cancer. The newest NCCN Guidelines for Patients focuses on uterine cancers (endometrial cancer and a much rarer type called uterine sarcoma). Uterine cancers are by far the most prevalent type of gynecologic cancer, impacting more than 63,000 new women every year, nearly twice the number of ovarian and cervical cancer diagnoses combined. With more than 11,000 lives estimated to be lost in 2018, uterine cancers are one of the highest causes of cancer deaths for women. ${ }^{1}$ Despite these statistics, awareness and resources around this cancer site are in short supply. NCCN created this new book of guidelines for patients, funded through the NCCN Foundation, to help increase public knowledge of uterine cancers.

"The NCCN Guidelines for Patients answer a real need for people with cancer and their loved ones, who want a better understanding of the medical decisions facing them," said Robert W. Carlson, MD, Chief Executive Officer, NCCN. "These guidelines go 
Cont. from page xxviii. beyond a basic overview, to truly explain, in plain language, what experts agree are the best, most up-to-date treatment approaches."

Endometrial cancer survivor Colleen Johnson, $\mathrm{PhD}$, understands that need firsthand. When she was diagnosed 6 years ago, the only information she could find came from a medical textbook.

"I wanted to leave my doctor's office with something in writing that explained what I was about to go through, to help me start reconciling what was going to happen," Johnson explained. "Instead, I spent hours searching the internet, living in intense fear that I would miss the one thing that would make the difference between life and death. I turned to a medical textbook, which just raised my anxiety levels even further. Thanks to these new guidelines from NCCN, women who are diagnosed with endometrial cancer or uterine sarcoma will finally have a good, solid source of accessible information about their cancer."

The NCCN Guidelines for Patients feature translated content from the NCCN Clinical Practice Guidelines in Oncology (NCCN Guidelines), in an easy-to-understand format that includes a glossary of terms and medical illustrations. The NCCN Guidelines are based on the latest evidence and consensus from a multidisciplinary team that includes surgeons, radiation oncologists, pathologists, and other experts from across the leading academic cancer centers that comprise NCCN. Both the clinical guidelines and their patient-focused counterparts are available free-of-charge for noncommercial use at NCCN.org or by app. The NCCN Guidelines for Patients will also be available in printed format soon through Amazon for a nominal fee.

"It's very useful for patients to be aware of what to expect from their treatment, based on state-of-the-art consensus," said Nadeem Abu-Rustum, MD, Memorial Sloan Kettering Cancer Center, Vice-Chair of the NCCN Guidelines Panel for Cervical, Uterine, and Vulvar Cancers. "I want people to know, if they're diagnosed with endometrial cancer, they should see a gynecologic oncologist, and not just rely on treatment from a general physician."

Dr. Abu-Rustum also conveyed that endometrial cancer can often be caught early. Women who experience abnormal staining or blood after reaching the age of menopause should talk to their doctor. When caught in the early stages, endometrial cancer is very treatable through minimally invasive surgery.

"We've made a lot of progress in the past 10 years, using technology to make surgery more precise," explained Dr. Abu-Rustum. "We've also expanded our understanding of the underlying biology for endometrial tumors. We're moving toward more incorporation of the molecular profile for increasingly accurate diagnosis and more targeted treatments."

The NCCN Guidelines for Patients: Uterine Cancer is endorsed by Facing Our Risk of Cancer Empowered (FORCE). NCCN Guidelines for Patients and NCCN Quick Guide sheets DO NOT replace the expertise and clinical judgment of the clinician.

The NCCN library of patient guidelines currently includes more than 40 different cancer types, including breast, colon, lung, and prostate cancers. The creation of these guidelines for uterine cancers was partially inspired by Johnson's role as an advocate and ultra-marathoner. Read more about her survival story at NCCN.org/colleen. To help

\section{References}

1. American Cancer Society ${ }^{\circledR}$ Cancer Statistics Center: https://cancerstatisticscenter.cancer.org. 